\title{
Wie Investoren mit hohen Staatsschulden umgehen
}

\author{
Julian Johs
}

\section{Relevanz}

Die Tragbarkeit der Staatsverschuldung ist begrenzt. Sind die Schulden hoch, ist nichts so wichtig wie die Glaubwürdigkeit der Finanzpolitik. Selbst überschuldete Länder können das Vertrauen der Investoren gewinnen, wenn sie eine Wende einleiten. Selbst bei hoher Verschuldung sind sinkende Schuldenquoten ein starkes Signal, dass die Finanzen unter Kontrolle sind und die Staaten ihre Schulden vollständig bedienen wollen. Die Wahrscheinlichkeit für einen erwarteten Zahlungsausfall sinkt, und damit die Risikoprämien. Fallende Zinsen erleichtern zudem eine nachhaltige Konsolidierung. Wenn jedoch beim selben hohen Schuldenstand die Verschuldung weiter zunimmt, ist das Vertrauen der Investoren schnell verloren.

\section{Quelle}

Bassanetti, Antonio, Carlo Cottarelli und Andrea F. Presbitero (2019), Lost and Found: Market Access and Public Debt Dynamics, Oxford Economic Papers 71, 445-471.

Fast alle Staaten haben Schulden. Viele haben große Probleme damit. Während und nach der Wirtschaftskrise sind die Schuldenstände, gemessen am BIP, stark angestiegen. Wenn die Investoren Zweifel an der Tragbarkeit der Staatsschulden bekommen, steigen die Risikoprämien rasant an. Die steigenden Zinsen

J. Johs $(\bowtie)$

WPZ Research, Wien, Österreich

E-Mail: julian.johs@wpz-research.com

(C) Der/die Autor(en) 2021 
können das Budget vollends in Schieflage bringen. Es droht ein Finanzierungsstopp. Einige hochverschuldete europäische Länder wie z. B. Griechenland und Irland haben den Zugang zum Kapitalmarkt ganz verloren. Wie können hoch verschuldete Staaten, die von einer Krise gebeutelt werden, den Zugang zum Kapitalmarkt erhalten oder wiederherzustellen? Worauf kommt es an?

Um das herauszufinden, untersuchen die italienischen Forscher Antonio Bassanetti, Carlo Cottarelli und Andrea Presbitero eine Vielzahl von Fällen mit akuten Schuldenproblemen von Staaten. Ein Schuldenproblem umfasst alle Formen von Zahlungsschwierigkeiten wie Verzüge bei Zins- und Tilgungszahlungen, Umschuldungen und Stundungen. In ihrer Untersuchung verwenden sie zwei Datensätze des Internationalen Währungsfonds für Industrie- als auch Schwellenländer. Diese Daten beziehen sich auf die Jahre 1970 bis 2014 und enthalten Schuldenstände, Budgetdefizite und Wachstumsraten sowie weitere Informationen. Die empirische Untersuchung beschränkt sich allerdings nur auf die Daten der Schwellenländer.

Der zweite Datensatz enthält Daten zum Verlust des Kapitalmarktzugangs. Sie beziehen sich auf jene Fälle, in denen die betroffenen Staaten plötzlich deutlich weniger Anleihen begeben oder Kredite aufnehmen können, oder Restrukturierungen bzw. teilweise Nichtzahlung von Schulden ankündigen müssen. Die Daten erfassen von 1990 bis 2013 über 44 Länder und enthalten ca. 50 Episoden, in denen Staaten keinen Marktzugang mehr hatten.

Ein Großteil der bisherigen Literatur erklärt das Entstehen von Schuldennotfällen hauptsächlich durch hohe Schuldenquoten. Die Autoren vermuten aber, dass die Investoren nicht nur auf den aktuellen Schuldenstand, sondern auch auf die Entwicklung in den vorangehenden Jahren schauen. Deutlich steigende Schuldenquoten könnten potenzielle Geldgeber als Hinweis verstehen, dass die betreffenden Staaten ihre Finanzen nicht mehr unter Kontrolle haben. Sinkende Schuldenquoten schaffen dagegen Vertrauen, dass sich die Staaten zu einem konsequenten Schuldenabbau verpflichtet haben und bereit sind, ihre Schulden vollständig zu bedienen.

Stark steigende Schuldenquoten interpretieren die Investoren tendenziell als fiskalischen Kontrollverlust. Sinkende Schuldenquoten schaffen hingegen Vertrauen, dass der Staat seine Schulden vollständig bedienen wird.

Die Forscher stellen fest, dass Schuldenprobleme in den 80er und frühen 90er Jahren besonders häufig waren. Danach ging die Zahl der Staaten mit Problemen zurück. Sie zählen 115 Episoden mit akuten Schuldenproblemen. In mehr als $90 \%$ der Fälle lagen hohe Auslandsschulden vor. In vier Fünftel der Notfälle gingen zwei Jahre mit steigender Schuldenquote voraus. 
Die Forscher gehen also davon aus, dass beim selben Schuldenstand die Wahrscheinlichkeit für den Eintritt eines Problemszenarios deutlich höher ausfällt, wenn zuvor die Schuldenentwicklung stark zunehmend war. Tatsächlich ergeben ihre Schätzungen: Eine Steigerung der Schuldenquote um zehn Prozentpunkte in den zwei vorangehenden Jahren ist verbunden mit einer um 1.3 Prozentpunkte höheren Wahrscheinlichkeit für ein Schuldenproblem. Dabei beträgt die durchschnittliche Wahrscheinlichkeit von akuten Schuldenproblemen $3.8 \%$.

Eine Steigerung der Schuldenquote um zehn Prozentpunkte in den zwei vorangehenden Jahren ist verbunden mit einer um 1.3 Prozentpunkte höheren Wahrscheinlichkeit für ein Schuldenproblem.

Die Forscher bestätigen auch die bisherigen Erkenntnisse, wonach die absolute Höhe der Schuldenquote einen wichtigen Einfluss auf die Problemanfälligkeit hat: Eine um 10 Prozentpunkte höhere Schuldenquote hängt mit einer durchschnittlich um 0.3 Prozentpunkte höheren Problemwahrscheinlichkeit zusammen. Dabei fällt das Risiko umso höher aus, je höher die Schuldenquote bereits ist. Abb. 1 zeigt das Zusammenspiel von Höhe und Entwicklung der Schuldenquote. Die oberste Kurve zeigt, dass Staaten nach einem starken Anstieg der Schuldenquote eine wesentlich höhere Ausfallswahrscheinlichkeit aufweisen, während

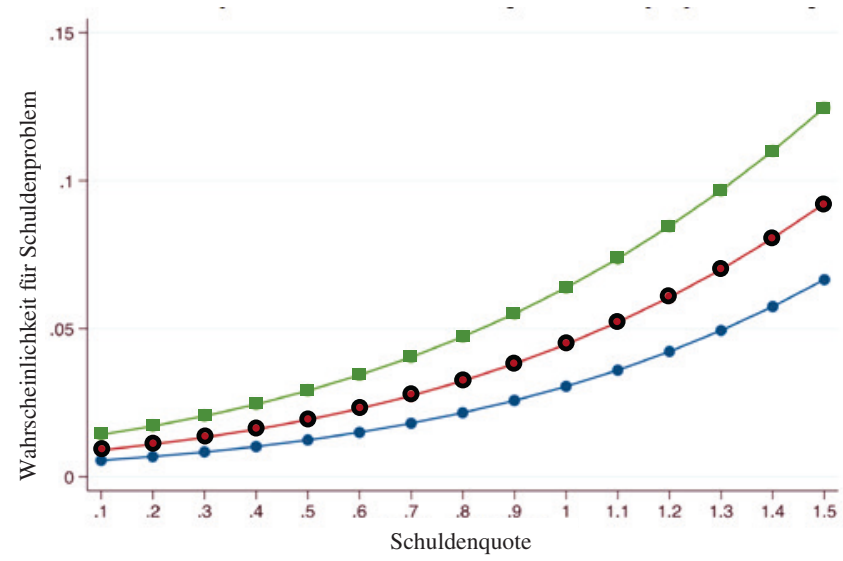

Schuldenrückgang um 5 Prozentpunkte

Schuldenquote unverändert

Schuldenzuwachs um 5 Prozentpunkte

Abb. 1 Wahrscheinlichkeit für einen Zahlungsausfall auf Staatsschulden 
Länder mit vorgängig fallenden Schuldenquoten (unterste Kurve) ein wesentlich geringeres Risiko aufweisen.

Wie steht es mit den Chancen, nach einer bereits eingetretenen Staatsinsolvenz wieder neues Vertrauen der Investoren und damit neuen Zugang zum Kapitalmarkt zu erlangen? Frühere Studien konzentrieren sich auch hier auf die Höhe der Schuldenquote. Die Wissenschaftler zeigen wiederum, dass nicht nur die Schuldenhöhe zählt. In der Hälfte der Fälle erhalten auch Länder mit sehr hohen Schuldenquoten wieder neuen Kapitalmarktzugang. Es ist also nicht zwingend nötig, dass Staaten ihre Schulden bereits reduziert haben müssen, bevor sie wieder am Markt teilnehmen können. Wiederum vermuten die italienischen Ökonomen, dass die Dynamik der Schulden eine wesentliche Rolle spielt. Selbst bei hohem Ausgangsniveau sendet eine sinkende Schuldenquote ein starkes Signal an den Markt, dass der Staat seine Finanzen unter Kontrolle gebracht und sich einer vollständigen Rückzahlung verpflichtet hat. Für die potenziellen Käufer von Staatsanleihen wirkt die Anlage daher sicherer. Steigt hingegen die Schuldenquote weiter an, ist das Vertrauen verloren. Die Investoren befürchten erst recht einen neuen Zahlungsausfall und verweigern neue Darlehen. Der Kapitalmarktzugang bleibt versperrt.

Die empirische Untersuchung bestätigt die Vermutung: Eine Senkung der Schuldenquote um zehn Prozentpunkte in den zwei vorangehenden Jahren ist verbunden mit einer durchschnittlich um 5.6 Prozentpunkte höheren Wahrscheinlichkeit, dass der Wiedereintritt in den Kapitalmarkt gelingt. Die durchschnittliche Wahrscheinlichkeit für neuen Kapitalmarktzugang beträgt $13.8 \%$. Die absolute Höhe der Schuldenquote selbst hängt nicht signifikant mit der Wahrscheinlichkeit für den Wiedereintritt zusammen.

Eine Senkung der Schuldenquote um zehn Prozentpunkte in den zwei vorangehenden Jahren geht einher mit einer um 5.6 Prozentpunkte höheren Wahrscheinlichkeit, dass der Wiedereintritt in den Kapitalmarkt gelingt.

Selbst ein hoher Schuldenstand muss nicht unbedingt eine Kapitalflucht auslösen. Sinkende Schuldenstände sind ein starkes Signal, dass die Staatsfinanzen unter Kontrolle sind. Indem überschuldete Staaten eine Wende einleiten, können sie das Vertrauen der Finanzmärkte wiederherstellen. Die Investoren revidieren die Ausfallswahrscheinlichkeit nach unten und fordern geringere Risikoprämien. Niedrige Zinsen erleichtern die allmähliche Konsolidierung des Staatshaushalts und reduzieren den Zwang zu abrupten Steuererhöhungen und Ausgabenkürzungen. So können die Staaten einer Negativspirale von zunehmenden Schulden, Vertrauensverlust, steigenden Zinsen und noch größerer Schieflage der Staatsfinanzen entkommen. 
Open Access Dieses Kapitel wird unter der Creative Commons Namensnennung 4.0 International Lizenz (http://creativecommons.org/licenses/by/4.0/deed.de) veröffentlicht, welche die Nutzung, Vervielfältigung, Bearbeitung, Verbreitung und Wiedergabe in jeglichem Medium und Format erlaubt, sofern Sie den/die ursprünglichen Autor(en) und die Quelle ordnungsgemäß nennen, einen Link zur Creative Commons Lizenz beifügen und angeben, ob Änderungen vorgenommen wurden.

Die in diesem Kapitel enthaltenen Bilder und sonstiges Drittmaterial unterliegen ebenfalls der genannten Creative Commons Lizenz, sofern sich aus der Abbildungslegende nichts anderes ergibt. Sofern das betreffende Material nicht unter der genannten Creative Commons Lizenz steht und die betreffende Handlung nicht nach gesetzlichen Vorschriften erlaubt ist, ist für die oben aufgeführten Weiterverwendungen des Materials die Einwilligung des jeweiligen Rechteinhabers einzuholen.

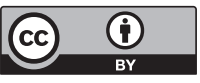

